\title{
Bone mesenchymal stromal cells exhibit functional inhibition but no chromosomal aberrations in chronic myelogenous leukemia
}

\author{
JIEQIONG XIE ${ }^{1}$, JIADI CHEN ${ }^{2}$, BIN WANG $^{1},{\text { XUCHUN } \mathrm{HE}^{3} \text { and HUIFANG HUANG }}^{1}$ \\ ${ }^{1}$ Central Laboratory and ${ }^{2}$ Fujian Institute of Hematology, Fujian Provincial Key Laboratory of Hematology, \\ Fujian Medical University Union Hospital, Fuzhou, Fujian 350001; ${ }^{3}$ Department of Medical Technology, \\ Fujian Health Career Technical College, Fuzhou, Fujian 350101, P.R. China
}

Received February 20, 2018; Accepted September 6, 2018

DOI: $10.3892 /$ ol.2018.9681

\begin{abstract}
Chronic myelogenous leukemia (CML) is a myeloproliferative neoplasia characterized by the presence of the Philadelphia $(\mathrm{Ph})$ chromosome in hematopoietic cells (HCs). As one of the most important components of the bone marrow microenvironment (BMM), bone mesenchymal stromal cells (BMSCs) are critical in the development of leukemia and essential in the regulation of hematopoiesis. However, little is known regarding the alterations of BMSCs in CML. The current study performed Cell Counting Kit- 8 and colony-forming unit fibroblast assays to evaluate the proliferative ability of BMSCs. The percentage of senescent BMSCs was evaluated by a senescence-associated $\beta$-galactosidase staining assay. Subsequently, a long-term culture-initiating cell assay was designed to explore the HC-supporting capacity of the BMSCs. Furthermore, cytogenetics were detected by conventional cytogenetic analysis and fluorescence in situ hybridization analysis. The current results revealed that CML-BMSCs exhibited decreased cell proliferation and impaired HC-support capacity, as well as increased susceptibility to senescence. No chromosomal aberrations, including the absence of the $\mathrm{Ph}$ chromosome, were noted in all CML-BMSCs. In conclusion, the current study demonstrated functional inhibition of CML-BMSCs; however, no signs of chromosomal aberrations were observed, thereby providing insight into the changes occurring in the CML-BMM.
\end{abstract}

\section{Introduction}

It is generally accepted that chronic myelogenous leukemia $(\mathrm{CML})$ is caused by a reciprocal translocation

Correspondence to: Professor Huifang Huang, Central Laboratory, Fujian Medical University Union Hospital, 29 Xinquan Road, Fuzhou, Fujian 350001, P.R. China

E-mail: huanghuif@126.com

Key words: chronic myelogenous leukemia, bone mesenchymal stromal cells, bone marrow microenvironment, functional inhibition, cytogenetics of chromosomes 9 and 22 termed the Philadelphia $(\mathrm{Ph})$ chromosome which results in the formation of the BCR-ABL fusion gene in primitive hematopoietic cells (HCs) (1). It has previously been reported that the BCR-ABL fusion gene initially appears in one single $\mathrm{HC}$, which subsequently induces a growth advantage for the cell and leads to excess multiplication (2). Over-accumulation of CML-HCs produces excessive amounts of granulocyte colony-stimulating factor, which downregulates the expression of C-X-C motif chemokine ligand 12 (CXCL12) in the bone marrow microenvironment (BMM) of CML. This downregulation of CXCL12 results in a reduction of the homing and retention of HCs and an increase in the number of CML-HCs in peripheral blood (3). Due to the genome instability in CML, CML-HCs are affected by accumulation of point mutations and chromosomal aberrations, which eventually facilitate blastic transformation $(4,5)$.

Within a decade, CML has progressed from a fatal disease to a disorder that can be controlled by lifelong oral medication (1). Use of BCR-ABL tyrosine kinase inhibitors has markedly improved the treatment of CML (6). However, drug resistance has become increasingly prominent in recent years (7). Previous studies have focused on drug combination (8) and protective effects of the BMM $(9,10)$, whereas the current study aims to provide a new perspective by investigating the changes that occur in the CML-BMM.

The BMM not only protects HCs from drugs but also participates in the development of leukemia $(9,10)$. As one of the main components of BMM, bone mesenchymal stromal cells (BMSCs) serve an indispensable role in hematopoiesis regulation (11). Previous studies have demonstrated that BMSCs derived from patients with myelodysplastic syndromes (MDS) and acute myeloid leukemia (AML) exhibit biological function impairment and a higher inclination to senescence compared with BMSCs derived from healthy donors (HDs) (12-14). In addition, genetic aberrations were detected in HCs in approximately $20 \%$ of patients with AML and $12 \%$ of patients with MDS (15). To the best of our knowledge, previous research concerning CML has mainly focused on malignant $\mathrm{HCs}$, with less focus on CML-BMSCs. This prompted the current study to analyze the biological characteristics and genetic alterations 
of the CML-BMM in order to explore its role in hematopoietic dysfunction.

\section{Materials and methods}

Patients and samples. A total of 6 healthy donors (HDs) (3 male, 3 female, 28-44 years of age, with a median age of 39 years) without malignant hematologic disease and 15 patients with CML diagnosed according to the 2016 World Health Organization criteria (16), were included in the current study. All patients and HDs were hospitalized at Fujian Medical University Union Hospital (Fuzhou, China) from August 2016 to March 2017. Patient clinical characteristics are summarized in Table I. Fresh heparinized bone marrow samples were obtained from the anterior superior iliac spine based on donors written informed consent. The current study was approved by the Ethical Committee of Fujian Medical University Union Hospital.

Isolation and culture of BMSCs. BMSCs were isolated from bone marrow aspirate by density gradient centrifugation $\left(500 \mathrm{x} \mathrm{g}, 30 \mathrm{~min}\right.$ at $25^{\circ} \mathrm{C}$ ) using Ficoll-Paque Plus (TBD, Tianjin, China, http://www.tbdscience.com/). Mononuclear cells were cultured with Dulbecco's modified Eagle's-low glucose medium (DMEM) (Hyclone; GE Healthcare, Logan, UT, USA) containing 10\% fetal bovine serum (Gibco; Thermo Fisher Scientific, Inc., Waltham, MA, USA) at $37^{\circ} \mathrm{C}$ under humidified atmosphere of $5 \% \mathrm{CO}_{2}$. After 24-48 h, the culture medium was discarded to remove non-adherent cells. Half of the medium was renewed every 3-4 days. When confluency reached 70-80\%, the cells were detached with $0.25 \%$ trypsin EDTA (Hyclone; GE Healthcare, Logan, UT, USA) for subculture.

Cell surface markers of BMSCs. Cell surface antigens were analyzed using a flow cytometer (Beckman Coulter, Inc., Brea, CA, USA). BMSCs at passage 3 were harvested and adjusted to $1 \times 10^{5}$ cells per tube. A total of six tubes were separately resuspended with $100 \mu 1$ phosphate buffer solution (PBS) (Hyclone; GE Healthcare) and labeled with $5 \mu 1$ monoclonal antibodies per $1 \times 10^{5}$ cells for $30 \mathrm{~min}$ in the dark at $4^{\circ} \mathrm{C}$ as follow: anti-CD45-phycoerythrin (PE) (12-0459-41), anti-CD34-PE (12-0349-41), anti-CD73-PE (12-0739-41), anti-CD90-PE (12-0909-41), anti-CD105-PE (12-1057-41) (all monoclonal antibodies 1:20 dilution, eBiosciences; Thermo Fisher Scientific Inc.). Species and isotype matched antibodies served as controls. Flow cytometric data analysis was made by Cytomics FC 500 analysis software (CXP 2.0, Beckman Coulter).

Colony-forming unit fibroblast (CFU-F) assay. BMSCs at passage 3 were detached with trypsin EDTA (Hyclone; GE Healthcare) and plated at a low density $\sim 1 \times 10^{2} / \mathrm{ml}$ onto a fresh culture dish. The cells were cultured for 14 days at $37^{\circ} \mathrm{C}$ under humidified atmosphere of $5 \% \mathrm{CO}_{2}$ in DMEM (Hyclone; GE Healthcare) and half of the medium was renewed every 4 days. The cells were then fixed with $4 \%$ paraformaldehyde for $20 \mathrm{~min}$ and stained with Giemsa for $30 \mathrm{~min}$ at room temperature. The stained cells were observed under a Nikon 7S-100 light microscope (Nikon Corporation, Tokyo, Japan) and colonies including $\geq 50$ cells were scored as a CFU-F.
Table I. Demographics and clinical characteristics of patients with chronic myelogenous leukemia included in the current study.

\begin{tabular}{lcc}
\hline Characteristics & No. & $\%$ \\
\hline Age, years & 45 & \\
Range & $24-67$ & \\
Sex & & 53.3 \\
Male & 8 & 46.7 \\
Female & 7 & 0 \\
Karyotype in HCs & & 100.0 \\
Normal & 0 & 100.0 \\
Aberrant & 15 & \\
FISH signals in HC & & \\
Normal & 0 & \\
Aberrant & 15 & \\
Median WBC, x10 $/ 1$ & 172.95 & \\
Range & $17.16-653.12$ & \\
Median ANC, x10 $/ 1$ & 94.17 & \\
Range & $14.71-575.98$ & \\
Median Hb, g/dl & 92.50 & \\
Range & $52.00-197.00$ & \\
Median PLTs, x $10^{9} / 1$ & 223.50 & \\
Range & $14.00-717.00$ & \\
\hline
\end{tabular}

HC, hematopoietic cell; FISH, fluorescent in situ hybridization; WBC, white blood cell; ANC, absolute neutrophil count; Hb, hemoglobin; PLTs, platelets.

Differentiation assay. BMSCs were harvested and seeded to 6 -well culture dishes at a concentration of $2 \times 10^{4} /$ well with DMEM (Hyclone; GE Healthcare). When the culture reached $80 \%$ confluency $\sim 5 \times 10^{5} /$ well, the medium was replaced with differentiation and induction medium from the StemPro Adipogenesis Differentiation kit, StemPro Osteogenesis Differentiation kit and the StemPro Chondrogenesis Differentiation kit (all kits from Invitrogen; Thermo Fisher Scientific, Inc.) for 2 weeks. The extent of adipogenesis, osteogenesis and chondrogenesis was observed by staining with oil red $\mathrm{O}$, alizarin red $\mathrm{S}$ and alcian blue, respectively according to the manufacturer's protocol at room temperature (all dyes from Beijing Solarbio Science \& Technology Co., Ltd., Beijing, China).

Cell Counting Kit-8(CCK8) assay to measure cell proliferation. BMSCs in an exponential phase of growth were collected and seeded into 96 -well plates at a concentration of $2 \times 10^{4} /$ well based on the CCK8 assay which was performed once a day for a total of 6 days. At $4 \mathrm{~h}$ after the cells were adhered, $10 \mu \mathrm{l} \mathrm{CCK} 8$ (Dojindo Molecular Technologies, Inc., Kumamoto, Japan) was added to each well. Following $2 \mathrm{~h}$ of incubation at $37^{\circ} \mathrm{C}$ under humidified atmosphere of $5 \% \mathrm{CO}_{2}$, densitometry was detected at the wavelength of $450 \mathrm{~nm}$ by a microplate reader (BioTek Instruments, Inc., Winooski, VT, USA). 
Senescence associated- $\beta$-galactosidase (SA- $\beta$-gal staining assay). BMSCs were harvested at passage 8 and seeded into a 6 -well plate at a concentration of $2 \times 10^{4}$ per well. An SA- $\beta$-gal staining kit (Beyotime Institute of Biotechnology, Haimen, China) was used to evaluate the percentage of senescent cells, according to the manufacturer's protocol. Subsequently, the plates were incubated for $12 \mathrm{~h}$ at $37^{\circ} \mathrm{C}$ under humidified atmosphere but without $\mathrm{CO}_{2}$. The percentage of blue-stained senescent cells per 500 cells was calculated in at least 10 random fields using a Nikon 7S-100 light microscope.

Long-term culture-initiating cell (LTC-IC) assay. An LTC-IC assay was conducted to detect the hematopoietic support ability of BMSCs. Firstly, BMSCs layers were seeded with $10 \mu \mathrm{l} / \mathrm{ml}$ mitomycin-c (Sigma-Aldrich) for $2 \mathrm{~h}$ at $37^{\circ} \mathrm{C}$ in humidified atmosphere of $5 \% \mathrm{CO}_{2}$. Then, HDs-HCs sorted using the CD34 MicroBead kit (Miltenyi Biotec Inc., Cambridge, MA, USA), were seeded onto the BMSC layers with MyeloCult H5100 medium (Stemcell Technologies, Inc., Vancouver, $\mathrm{BC}$, Canada) for 5 weeks. Co-cultured $\mathrm{CD} 34^{+}$cells were then collected and cultured in MethoCult H4431 medium (Stemcell Technologies, Inc.) at a concentration of $1 \times 10^{3} / \mathrm{ml}$ per dish for a further 14 days. Colony forming units of granulocyte/monocyte (CFU-GM) ( $\geq 40$ cells), colony forming units of erythroid (CFU-E) ( $\geq 50$ cells) and burst forming units of erythroid (BFU-E) ( $\geq 300$ cells) were counted under the Nikon 7S-100 light microscope.

Conventional cytogenetic (CC) analysis and fluorescent in situ hybridization (FISH) analysis of HCs and BMSCs. Cytogenetics of HCs and BMSCs were evaluated by CC analysis and FISH analysis. Briefly, the cells were maintained at metaphase by adding colcemide (Gibco; Thermo Fisher Scientific, Inc.) at a final concentration of $0.2 \mu \mathrm{g} / \mathrm{ml}$. The air-drying method was used to prepare the glass slides. CC analysis was carried out by R banding (17). Karyotypic abnormalities were described in detail in accordance with the International System for Human Cytogenetic Nomenclature (2013) (18). A BCR-ABL probe (GP Medical Technologies, Ltd., Beijing, China, http://www.gpmedical.com.cn/) was used according to the manufacturer's protocol to measure BCR-ABL translocations by FISH analysis. ABL gene on chromosome 22 is marked with the red signal, BCR gene on chromosome 9 with the green signal, and the hybridization signal of BCR-ABL fusion gene shows the yellow signal. The results were detected by an Olympus BX51 fluorescence microscope (Olympus Corporation, Tokyo, Japan).

Statistical analysis. Quantitative data are presented as mean \pm standard deviation. A two-sided unpaired Student's t-test was applied to compare two different groups using SPSS 20.0 (IBM Corp., Armonk, NY, USA). $\mathrm{P}<0.05$ was considered to indicate a statistically significant difference.

\section{Results}

Identification of BMSCs. Samples from HDs and patients with CML were collected to verify whether isolated cells met the minimal definition criteria for BMSCs proposed by the International Society for Cellular Therapy (ISCT) (19).
As demonstrated in Fig. 1A, the isolated cells from HDs were negative for $\mathrm{HC}$ antigens, including CD34 and CD45 $(<10 \%)$, but positive for CD73, CD90 and CD105 (>90\%), which was consistent with previous studies $(14,20)$, as well as CML-BMSCs (data not shown). A CFU-F assay also verified the isolated cells were BMSCs. As demonstrated in Fig. 1B, one single cell from HDs was able to form a visible colony at passage 3 and this characteristic could also be detected at passage 8 (data not shown) as well as CML-BMSCs (data not shown), indicating the CML-BMSCs did not lose self-renewal capacity before or at passage 8 . A differentiation assay revealed that HD-BMSCs at passage 3 exhibited the ability to undergoing multipotential differentiation as it could turned into three different shapes after being induced by the corresponding induction differentiation culture conditions as illustrated in Fig. 1C, which was still maintained at passage 6 (data not shown). Likewise, multipotential differentiation ability was also observed in CML-BMSCs (data not shown).

In summary, the adherent cells isolated were BMSCs, which possessed stable stem cell properties that provided sufficient reliability for subsequent experimentation.

BMSC culture and morphology. The amplification culture of HD-BMSCs and CML-BMSCs was measured. It was identified that it took 15 days on average, with a range of 7-30 days, for primary BMSCs to reach $80 \%$ confluency. Notably, it appeared to be harder for HD-BMSCs to adapt to culture conditions in vitro compared with CML-BMSCs. HD-BMSCs required a longer period of time (average of 20 days) to pass on to the next generation than CML-BMSCs (average of 10 days) at the first division. Both HD-BMSCs and CML-BMSCs grew at a faster rate after passage 1 (average of 3-7 days) and then slowed down in growth at passage 5 (average of 5-14 days).

The current study demonstrated that both HD-BMSCs and CML-BMSCs exhibited fibroblast-like shape at the early passage such as passage 3 (Fig. 1D a-b). As the passages increased, a higher number of CML-BMSCs grew larger and more irregular in shape compared with HD-BMSCs at passage 5 (Fig. 1D c-d).

Impaired growth kinetics of CML-BMSCs. To evaluate the growth rate of HD-BMSCs and CML-BMSCs, a CCK8 assay was performed. Over the 6-day culture period, CML-BMSCs grew at a significantly slower speed compared with HD-BMSCs between days 3 and $6(\mathrm{P}<0.05$; Fig. 2A). Furthermore, by seeding 200 cells per dish, a CFU-F assay was performed to assess the self-renewal ability of BMSCs. CML-BMSCs produced a significantly lower number of CFU-Fs compared

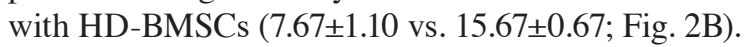

Increased cellular senescence of CML-BMSCs. The current study identified that BMSCs gradually changed from a fibroblast-like shape into a large and irregular shape, which was previously described as a typical senescence characteristic (21). In view of this finding, SA- $\beta$-gal stain was used to assess the percentage of BMSCs undergoing senescence. As demonstrated in Fig. 2C, the percentage of senescent HD-BMSC $(22.33 \pm 1.61 \%)$ was significantly decreased compared with the percentage of senescent CML-BMSC (60.17 $\pm 2.66 \%)$. 

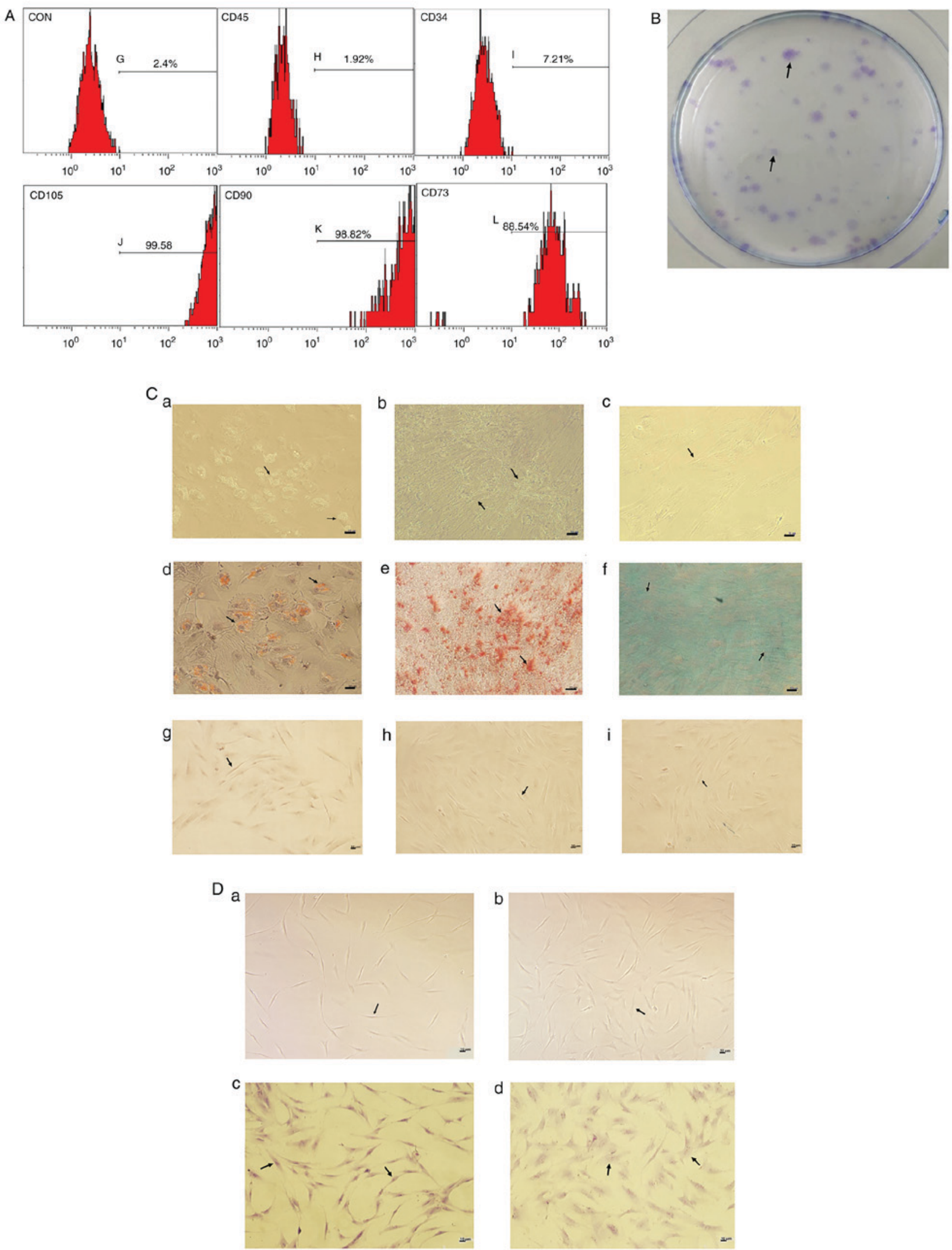

Figure 1. Identification of BMSCs. (A) BMSCs were negative for CD45 (1.92\%) and CD34 (7.21\%) but positive for CD73 (88.54\%), CD90 (98.82\%) and CD105 (99.58\%). (B) By seeding 1,000 cells in a $10 \mathrm{~mm}$ dish, BMSCs demonstrated the ability to form a visible colony unit as indicated by the arrows. (C) BMSCs exhibited the capacity to differentiate to $(\mathrm{C}-\mathrm{a})$ adipogenic cells, (C-b) osteogenic cells and $(\mathrm{C}-\mathrm{c})$ chondrocytes as indicated by the arrows. The corresponding staining results are presented in in (Cd-f), (C-d) adipogenic cells stained with oil red $\mathrm{O},(\mathrm{C}-\mathrm{e})$ osteogenic cells stained with alizarin red $\mathrm{S}$ and $(\mathrm{C}-\mathrm{f})$ chondrocytes stained with alcian blue are indicated by the arrows. (Cg-i) Negative controls were undifferentiated BMSCs respectively stained with $(\mathrm{Cg})$ oil red $\mathrm{O},(\mathrm{Ch})$ alizarin red $\mathrm{S}$ and (Ci) alcian blue which were indicated by the arrows. (D-a) HD-BMSCs and (D-b) CML-BMSCs at passage 3 exhibited fibroblast-like morphology as indicated by the arrows. (D-c) Certain HD-BMSCs at passage 5 grew excessively large and exhibited an irregular shape as indicated by the arrows. (D-d) Compared with HD-BMSCs, a higher number of CML-BMSCs at passage 5 grew excessively larger and exhibited an irregular shape as indicated by the arrows. Black lines represent $10 \mu \mathrm{m}$. Magnification, x100. CON, control; BMSCs, bone mesenchymal stromal cells; HD, healthy donor; CML, chronic myelogenous leukemia. 
A

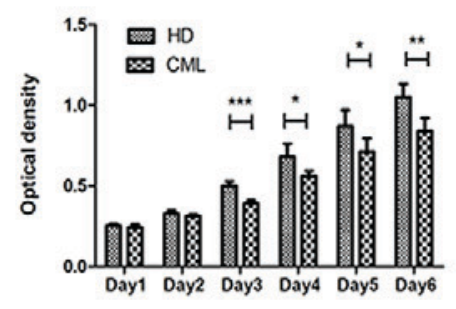

$\mathrm{C}$
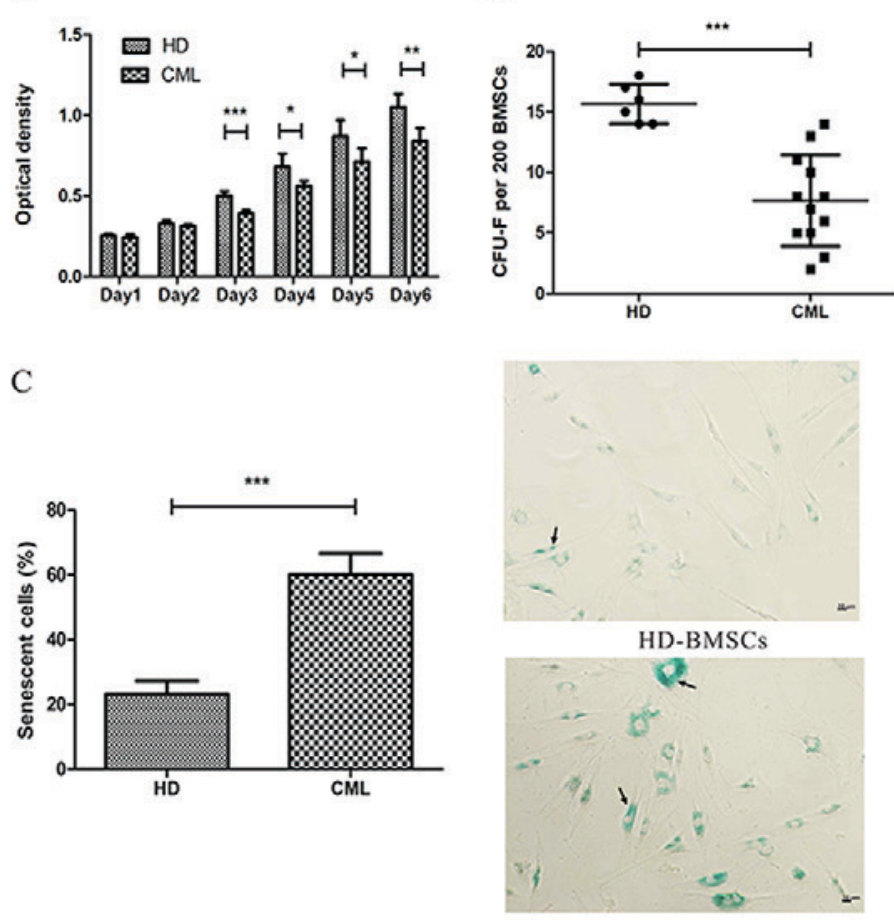

CML-BMSCs

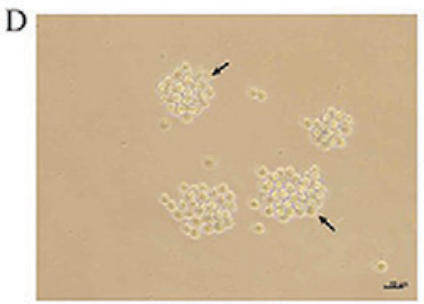

CFU-GM

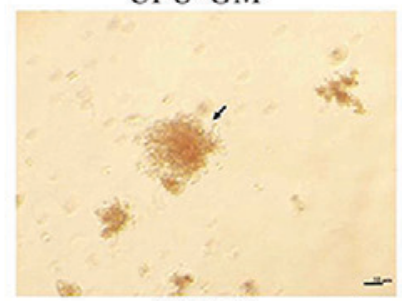

CFU-E

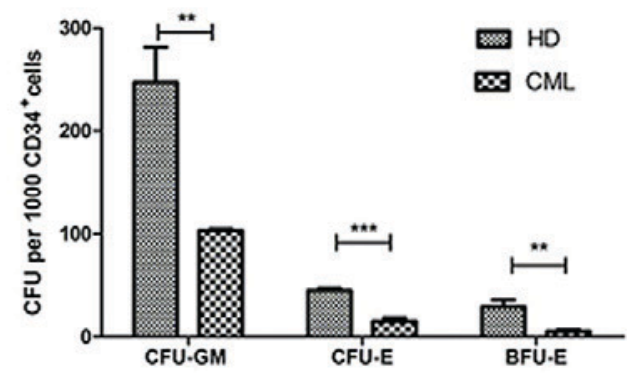

Figure 2. Biological characteristics of BMSCs. (A) Cell proliferation capacity of CML-BMSCs ( $\mathrm{n}=5$ ) was significantly impaired compared with that of HD-BMSCs ( $n=5$ ) on days 3 to 6 . (B) Compared with HD-BMSCs $(n=6)$ the CFU-F number of CML-BMSCs $(n=12)$ was significantly lower $(15.67 \pm 0.67$ vs. $7.67 \pm 1.1)$. (C) The percentage of senescent CML-BMSCs was significantly higher compared with that of HD-BMSCs $(60.17 \pm 2.66$ vs. $22.33 \pm 1.61 \%)$. The senescent cells were stained blue as indicated by the arrows. Black lines represent $10 \mu \mathrm{m}$. Magnification, x100. (D) The hematopoietic support capacity was impaired in CML-BMSCs compared with that of HD-BMSCs. CFU-GM as indicated by the arrows (left panel) formed by CD34 ${ }^{+} \mathrm{HCs}$ following co-culture with CML-BMSCs was smaller than that co-culture with HD-BMSCs (the bigger CFU-GM as indicated by the arrows in right panel). CFU-E as indicated by the arrows appeared more in CD34+ $\mathrm{HCs}-\mathrm{CML}-\mathrm{BMSCs}$ co-culture system, while BFU-E as indicated by the arrows appeared more in CD34 ${ }^{+} \mathrm{HCs}-\mathrm{HD}-\mathrm{BMSCs}$ co-culture system (The picture from left to right top to bottom is as follows: Smaller CFU-GM, bigger CFU-GM, CFU-E and BFU-E as indicated by the arrows). The numbers of CFU-GM, CFU-E and BFU-E formed by CD34 ${ }^{+} \mathrm{HCs}$ were decreased when cultured with CML-BMSCs as opposed to HD-BMSCs (histogram). Black lines represent $10 \mu \mathrm{m}$. Magnification, $\mathrm{x} 400$. Data are presented as the mean \pm standard deviation. ${ }^{*} \mathrm{P}<0.05,{ }^{* * *} \mathrm{P} \leq 0.01,{ }^{* * * *} \mathrm{P} \leq 0.001$. BMSCs, bone mesenchymal stromal cells; CML, chronic myelogenous leukemia; HD, healthy donor; CFU-F, colony forming units of fibroblast; CFU-GM, colony forming units of granulocyte/monocyte; CFU-E, colony forming units of erythroid; BFU-E, burst forming units of erythroid. 
A
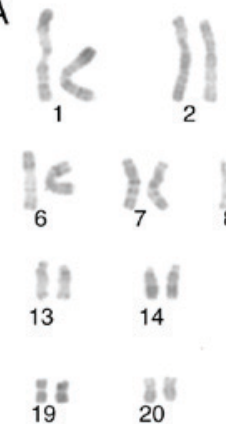

14

36
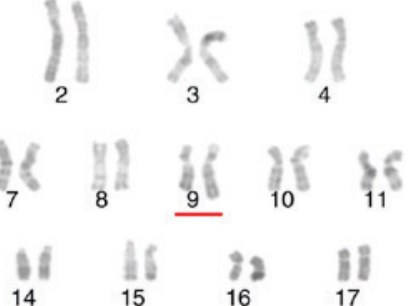

15

$21 \quad \underline{22}$

CML-HC

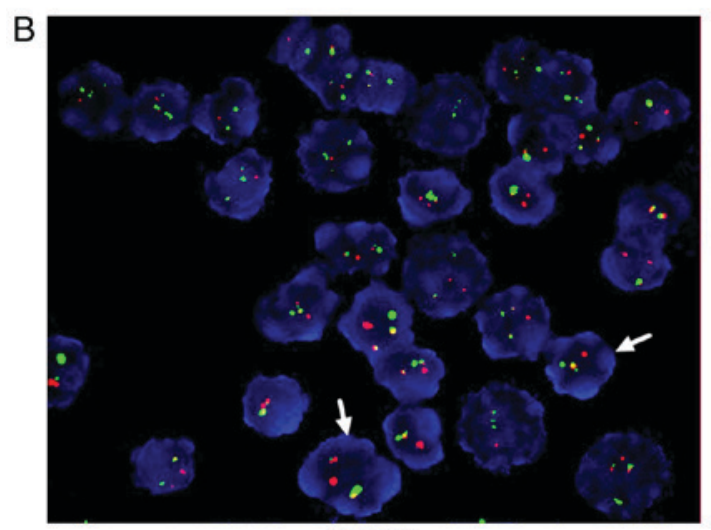

$\mathrm{CML-HC}$
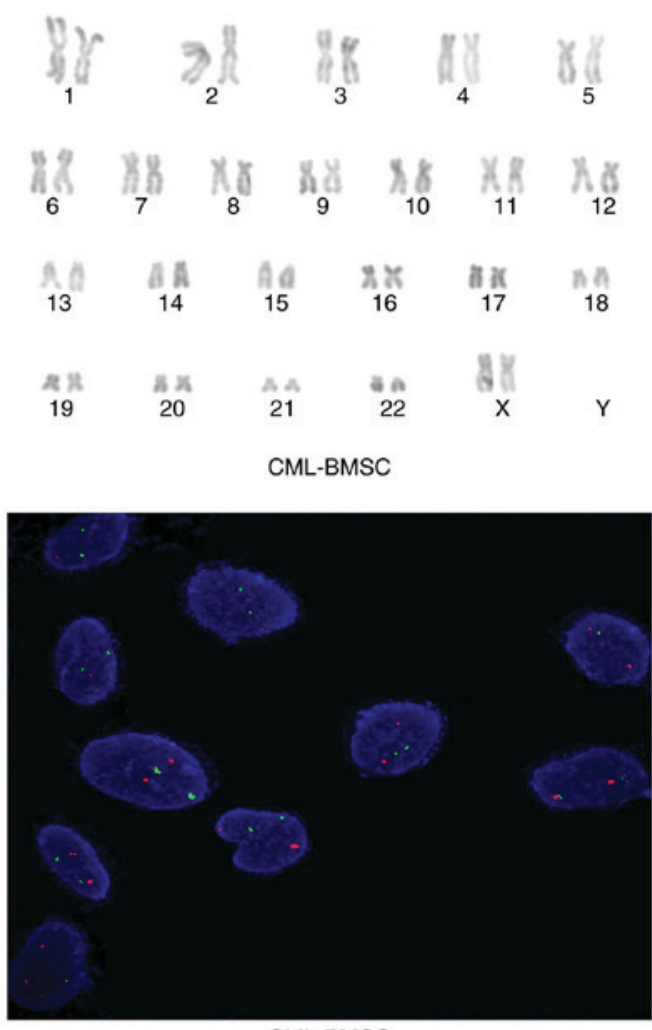

CML-BMSC

Figure 3. Cytogenetics of patients with CML. (A) Conventional cytogenetic analysis identified the Philadelphia chromosome in CML-HCs (left panel) and a normal karyotype in CML-BMSCs (right panel). (B) Fluorescent in situ hybridization analysis revealed yellow signals in CML-HCs, which represented the BCR-ABL fusion gene (left panel). The yellow signal was negative in CML-BMSCs (right panel). CML, chronic myelogenous leukemia; BMSCs, bone mesenchymal stromal cells; HCs, hematopoietic cells.

Diminished hematopoietic support capacity of CML-BMSCs. An LTC-IC assay was implemented to evaluate whether the hematopoietic support capacity of CML-BMSCs was impaired compared with that of HD-BMSCs. By counting the number of CFUs, the current study identified that the LTC-IC frequency (the total number of CFU-GMs, CFU-Es and BFU-Es) was 2.6-fold higher following co-culture with HD-BMSCs compared with co-culture with CML-BMSCs, as well as bigger size of CFU (Fig. 2D upper panel). In detail, CD34 ${ }^{+}$ HCs cultured with CML-BMSCs demonstrated decreased numbers of CFU-GM (103.25 \pm 2.87 vs. 267.25 \pm 67.54$)$, CFU-E $(15.25 \pm 6.24$ vs. $45.00 \pm 3.92)$ and BFU-E (5.50 \pm 2.65 vs. 29.25 \pm 12.31$)$ compared with those cultured with HD-BMSCs (Fig. 2D lower panel). The current results identified that CML-BMSCs exhibited a less effective hematopoietic support capability compared with HD-BMSCs.

Absence of BCR-ABL in CML-BMSCs. To gain further insight into CML-BMSCs, CC analysis and FISH analysis were used to explore cytogenetic alterations in BMSCs and HCs from 15 patients with CML and 6 HDs. The current study revealed that the $\mathrm{Ph}$ chromosome could be identified in all CML-HCs that were analyzed by CC. The FISH results demonstrated that all CML-HCs as indicated by the yellow hybridization signal. However, neither no chromosomal aberrations were identified in CML-BMSCs using $\mathrm{CC}$ analysis (Fig. 3A), no yellow signal was detected by FISH analysis (Fig. 3B and Table II). As expected, HCs and
BMSCs in all HDs exhibited a normal karyotype (data not shown).

\section{Discussion}

Previously, several studies have proposed a hypothesis that functional and genetic alterations of stromal cells would induce myeloid malignancies $(22,23)$. Therefore, the current study focused on BMSCs to explore whether their functional and genetic characteristics were altered in CML-BMM.

The current study identified that there were no significant differences in surface marker expression and the ability of multipotential differentiation between the adherent cells derived from HDs and patients with CML. Adherent cells from both HDs and patients with CML conformed to the minimal definition criteria for BMSCs proposed by the ISCT. In addition, the separation method used in the current study was confirmed to be reliable. The current study revealed that the growth rate of CML-BMSCs was impaired compared with that of HD-BMSCs. Previous studies have reported that impaired proliferation is not associated with cell cycle arrest or increased rates of apoptosis (20), but is associated with an increased rate of aging (14). The current study identified that CML-BMSCs were more susceptible to cellular senescence compared with HD-BMSCs. The promyelocytic leukemia gene plays a key role in the progress of BMSCs senescence (24). Cellular senescence in BMSCs 
Table II. Chromosomal aberrations in BMSCs.

\begin{tabular}{|c|c|c|c|c|}
\hline \multirow[b]{2}{*}{ Patient no. } & \multicolumn{2}{|c|}{ Karyotype } & \multicolumn{2}{|c|}{$\mathrm{BCR} / \mathrm{ABL}$ dual fusion } \\
\hline & $\mathrm{HCs}$ & BMSCs & $\mathrm{HCs}$ & BMSCs \\
\hline 1 & $46, X Y, t(9 ; 22)(q 34 ; q 11.2)[16]$ & $46, X Y[20]$ & + & - \\
\hline 2 & $46, X Y, t(9 ; 22)(q 34 ; q 11.2)[12]$ & $46, X Y[14]$ & + & - \\
\hline 3 & $46, X X, t(9 ; 22)(q 34 ; q 11.2)[20]$ & $46, X X[17]$ & + & - \\
\hline 4 & $46, X Y, t(9 ; 22)(q 34 ; q 11.2)[13]$ & $46, X Y[13]$ & + & - \\
\hline 5 & $46, X Y, t(9 ; 22)(q 34 ; q 11.2)[11]$ & $46, X Y[20]$ & + & - \\
\hline 6 & $46, X Y, t(9 ; 22)(q 34 ; q 11.2)[17]$ & $46, X Y[20]$ & + & - \\
\hline 7 & $46, X X, t(9 ; 22)(q 34 ; q 11.2)[19]$ & $46, X X[20]$ & + & - \\
\hline 8 & $46, X Y, t(9 ; 22)(q 34 ; q 11.2)[15]$ & $46, X Y[18]$ & + & - \\
\hline 9 & $46, \mathrm{XX}, \mathrm{t}(9 ; 22)(\mathrm{q} 34 ; \mathrm{q} 11.2)[18]$ & $46, X X[12]$ & + & - \\
\hline 10 & $46, X Y, t(9 ; 22)(q 34 ; q 11.2)[12]$ & $46, X Y[20]$ & + & - \\
\hline 11 & $46, \mathrm{XX}, \mathrm{t}(9 ; 22)(\mathrm{q} 34 ; \mathrm{q} 11.2)[20]$ & $46, \mathrm{XX}[13]$ & + & - \\
\hline 12 & $46, \mathrm{XX}, \mathrm{t}(9 ; 22)(\mathrm{q} 34 ; \mathrm{q} 11.2)[14]$ & $46, \mathrm{XX}[13]$ & + & - \\
\hline 13 & $44, \mathrm{XX}, \mathrm{t}(9 ; 22)(\mathrm{q} 34 ; \mathrm{q} 11.2)[16]$ & $46, X X[20]$ & + & - \\
\hline 14 & $46, X Y, t(9 ; 22)(q 34 ; q 11.2)[15]$ & $46, X Y[16]$ & + & - \\
\hline 15 & $46, X X, t(9 ; 22)(q 34 ; q 11.2)[20]$ & $46, X X[14]$ & + & - \\
\hline
\end{tabular}

HCs, hematopoietic cells; BMSCs, bone mesenchymal stromal cells.

may affect their hematopoietic support capacity (25). The current study demonstrated that the frequency of LTC-ICs following co-culture with HD-BMSCs was 2.6-fold higher compared with that of CML-BMSCs, indicating that CML-BMSCs exhibited an impaired hematopoietic support capacity compared with HD-BMSCs. Within the BMM in hematologic neoplasms, malignant HCs and healthy HCs are affected by exosomes, microRNAs, long non-coding RNAs and cytokines secreted by stromal cells (26-28). Among them, to the best of our knowledge, the research seem to focus more on the effects of cytokines on hematopoietic support capacity in BMM at present $(3,29)$. A limitation of the current study is that only the phenomenon of biological characteristics of CML-BMSCs were provided, while in-depth research on the mechanism of the impaired biological characteristics was not conducted. In the near future, the effects of cytokine changes on the impaired hematopoietic support capacity of CML-BMSCs may be investigated. It has previously been reported that malignant HCs may remodel and transform a healthy BMM into an inflammatory environment $(30,31)$. When exposed to an inflammatory environment in vivo, the epigenetics of BMSCs would be altered and functions of BMSCs would be impaired, including their hematopoietic support capacity $(12,13)$. A previous study demonstrated that the capacity of BMSCs to support healthy HCs was impaired, however they selectively promoted the proliferation of malignant HCs (32).

Growing evidence has demonstrated that genetic abnormalities exist in BMSCs derived from leukemia patients $(15,33)$. This suggests that a primary BMSC defect either causes or supports hematologic malignancy. This hypothesis challenges the popular theory that hematological malignancies originate exclusively from intrinsic genetic defects in HCs (34).
The current study did not detect any of the cytogenetic abnormalities in CML-BMSCs that have been identified in previous studies $(35,36)$. As previously mentioned, the current study has verified that the biological function of BMSCs was impaired when derived from patients with CML patients compared with that of HD-BMSCs. Functional inhibition has been demonstrated to be associated with DNA methylation in AML and MDS-derived BMSCs $(12,13)$. Abnormalities of DNA methylation may lead to gene instability $(37,38)$. Therefore, the current study assumed that BMSCs with altered biological functions may exhibit a potential risk of gene mutation. The accumulation of genetic mutations may eventually lead to chromosomal aberrations $(34,39)$. BMSCs and HCs may similarly be affected by a predisposing genetic defect and therefore contribute equally to the manifestation of CML (40).

Whether genetic stability of BMSCs could be maintained in vitro is of note. A previous study has revealed genetic material of BMSCs remains intact up to and including passage 9 (41). All BMSCs adopted in the current study for chromosomal analysis were within passage 5 . Therefore, it is likely the BMSCs used in the current study only acquired additional mutations at a very low frequency.

In summary, the current study performed a comprehensive analysis that demonstrated the function of CML-BMSCs was inhibited but no cytogenetic abnormalities were detected. The current study has provided further insight into the pathophysiology of the CML-BMM with the view of providing an improved understanding of BMSCs.

\section{Acknowledgements}

Not applicable. 


\section{Funding}

The present study was supported by the Fujian Medical University Professor Fund (grant no. JS14024) and Fujian Provincial Natural Fund (grant no. 2015J01472).

\section{Availability of data and materials}

The analyzed data are available from the corresponding author upon reasonable request.

\section{Authors' contributions}

JQX and HFH designed the experiments. JQX and JDC performed the laboratory work for this study. BW and $\mathrm{XCH}$ recruited the patients. BW participated in data analysis. $\mathrm{XCH}$ performed part of the laboratory work and drafted the corresponding part of the manuscript. JQX and HFH worked on the manuscript. All the authors have read and approved the final version of the manuscript.

\section{Ethics approval and consent to participate}

The Ethical Committee of Fujian Medical University Union Hospital (approval no. 2016KY053). Participants provided written informed consent for their inclusion in the present study.

\section{Patient consent for publication}

All patients provided written informed consent for the publication of their data and any associated images.

\section{Competing interests}

The authors declare that they have no competing interests.

\section{References}

1. Apperley JF: Chronic myeloid leukaemia.Lancet 385: 1447-1459, 2015.

2. Bruns I, Czibere A, Fischer JC, Roels F, Cadeddu RP, Buest S, Bruennert D, Huenerlituerkoglu AN, Stoecklein NH, Singh R, et al: The hematopoietic stem cell in chronic phase CML is characterized by a transcriptional profile resembling normal myeloid progenitor cells and reflecting loss of quiescence. Leukemia 23: 892-899, 2009.

3. Zhang B, Ho YW, Huang Q, Maeda T, Lin A, Lee SU, Hair A, Holyoake TL, Huettner C and Bhatia R: Altered microenvironmental regulation of leukemic and normal stem cells in chronic myelogenous leukemia. Cancer Cell 21: 577-592, 2012.

4. Penserga ET and Skorski T: Fusion tyrosine kinases: A result and cause of genomic instability. Oncogene 26: 11-20, 2007.

5. Melo JV and Barnes DJ: Chronic myeloid leukaemia as a model of disease evolution in human cancer. Nat Rev Cancer 7: 441-453, 2007.

6. Goldman JM and Melo JV: Targeting the BCR-ABL tyrosine kinase in chronic myeloid leukemia. N Engl J Med 344: 1084-1086, 2001.

7. Goldman JM and Melo JV: Chronic myeloid leukemia-advances in biology and new approaches to treatment. N Engl J Med 349: 1451-1464, 2003

8. Zhou H, Mak PY, Mu H, Mak DH, Zeng Z, Cortes J, Liu Q, Andreeff $\mathrm{M}$ and Carter BZ: Combined inhibition of $\beta$-catenin and Bcr-Abl synergistically targets tyrosine kinase inhibitor-resistant blast crisis chronic myeloid leukemia blasts and progenitors in vitro and in vivo. Leukemia 31: 2065-2074, 2017.

9. Jin L, Tabe Y, Konoplev S, Xu Y, Leysath CE, Lu H, Kimura S, Ohsaka A, Rios MB, Calvert L, et al: CXCR4 up-regulation by imatinib induces chronic myelogenous leukemia (CML) cell migration to bone marrow stroma and promotes survival of quiescent CML cells. Mol Cancer Ther 7: 48-58, 2008.
10. Schroeder T, Geyh S, Germing U and Hass R: Mesenchymal stromal cells in myeloid malignancies. Blood Res 51: 225-232, 2016.

11. Frenette PS, Pinho S, Lucas D and Scheiermann C: Mesenchymal stem cell: Keystone of the hematopoietic stem cell niche and a stepping-stone for regenerative medicine. Annu Rev Immunol 31: 285-316, 2013.

12. Geyh S, Rodriguez-Paredes $\mathrm{M}$, Jäger $\mathrm{P}$, Khandanpour $\mathrm{C}$, Cadeddu RP, Gutekunst J, Wilk CM, Fenk R, Zilkens C, Hermsen D, et al: Functional inhibition of mesenchymal stromal cells in acute myeloid leukemia. Leukemia 30: 683-691, 2016.

13. Geyh S, Oz S, Cadeddu RP, Fröbel J, Brückner B, Kündgen A, Fenk R, Bruns I, Zilkens C, Hermsen D, et al: Insufficient stromal support in MDS results from molecular and functional deficits of mesenchymal stromal cells. Leukemia 27: 1841-1851, 2013.

14. Zhao Y, Wu D, Fei C, Guo J, Gu S, Zhu Y, Xu F, Zhang Z, Wu L, Li X and Chang C: Down-regulation of Dicerl promotes cellular senescence and decreases the differentiation and stem cell-supporting capacities of mesenchymal stromal cells in patients with myelodysplastic syndrome. Haematologica 100: 194-204, 2015.

15. Blau O, Baldus CD, Hofmann WK, Thiel G, Nolte F, Burmeister T, Türkmen S, Benlasfer O, Schümann E, Sindram A, et al: Mesenchymal stromal cells of myelodysplastic syndrome and acute myeloid leukemia patients have distinct genetic abnormalities compared with leukemic blasts. Blood 118: 5583-5592, 2011.

16. Arber DA, Orazi A, Hasserjian R, Thiele J, Borowitz MJ, Le Beau MM, Bloomfield CD, Cazzola M and Vardiman JW: The 2016 revision to the World Health Organization classification of myeloid neoplasms and acute leukemia. Blood 127: 2391-2405, 2016.

17. Bernheim A: Cytogenomics of cancers: From chromosome to sequence. Mol Oncol 4: 309-322, 2010.

18. Simons A, Shaffer LG and Hastings RJ: Cytogenetic nomenclature: Changes in the ISCN 2013 compared to the 2009 Edition. Cytogenet Genome Res 141: 1-6, 2013.

19. Dominici M,Le Blanc K, Mueller I, Slaper-Cortenbach I, Marini F Krause D, Deans R, Keating A, Prockop Dj and Horwitz E: Minimal criteria for defining multipotent mesenchymal stromal cells. The International Society for cellular therapy position statement. Cytotherapy 8: 315-317, 2006.

20. Lopez-Villar O, Garcia JL, Sanchez-Guijo FM, Robledo C, Villaron EM, Hernández-Campo P, Lopez-Holgado N, Diez-Campelo M, Barbado MV, Perez-Simon JA, et al: Both expanded and uncultured mesenchymal stem cells from MDS patients are genomically abnormal, showing a specific genetic profile for the 5q-syndrome. Leukemia 23: 664-672, 2009.

21. Campisi J and d'Adda di Fagagna F: Cellular senescence: When bad things happen to good cells. Nat Rev Mol Cell Biol 8: 729-740, 2007.

22. Rupec RA, Jundt F, Rebholz B, Eckelt B, Weindl G, Herzinger T, Flaig MJ, Moosmann S, Plewig G, Dörken B, et al: Stroma-mediated dysregulation of myelopoiesis in mice lacking I kappa B alpha. Immunity 22: 479-491, 2005.

23. Schepers K, Pietras E, Reynaud D, Flach J, Binnewies M, Garg T, Wagers AJ, Hsiao EC and Passegué E: Myeloproliferative neoplasia remodels the endosteal bone marrow niche into a self-reinforcing leukemic niche. Cell Stem Cell 13: 285-299, 2013.

24. Fu S, Wei J, Wang G, Wang B, Wang Y, Lai X and Huang H: The key role of PML in IFN- $\alpha$ induced cellular senescence of human mesenchymal stromal cells. Int J Oncol 46: 351-359, 2015.

25. Walenda T, Bork S, Horn P, Wein F, Saffrich R, Diehlmann A, Eckstein V, Ho AD and Wagner W: Co-culture with mesenchymal stromal cells increases proliferation and maintenance of haematopoietic progenitor cells. J Cell Mol Med 14: 337-350, 2010.

26. Kumar B, Garcia M, Weng L, Jung X, Murakami JL, Hu X, McDonald T, Lin A, Kumar AR, DiGiusto DL, et al: Acute myeloid leukemia transforms the bone marrow niche into a leukemia-permissive microenvironment through exosome secretion. Leukemia 32: 575-587, 2018.

27. Barrera-Ramirez J, Lavoie JR, Maganti HB, Stanford WL, Ito C, Sabloff M, Brand M, Rosu-Myles M, Le Y and Allan DS: Micro-RNA profiling of exosomes from marrow-derived mesenchymal stromal cells in patients with acute myeloid leukemia: Implications in leukemogenesis. Stem Cell Rev 13: 817-825, 2017.

28. Shah MY, Ferracin M, Pileczki V, Chen B, Redis R, Fabris L, Zhang X, Ivan C, Shimizu M, Rodriguez-Aguayo C, et al: Cancer-associated rs6983267 SNP and its accompanying long noncoding RNA CCAT2 induce myeloid malignancies via unique SNP-specific RNA mutations. Genome Res 28: 432-447, 2018 . 
29. Hong DS, Angelo LS and Kurzrock R: Interleukin-6 and its receptor in cancer: Implications for translational therapeutics. Cancer 110: 1911-1928, 2007.

30. Hoggatt J, Kfoury Y and Scadden DT: Hematopoietic stem cell niche in health and disease. Annu Rev Pathol 11: 555-581, 2016.

31. Schepers K, Campbell TB and Passegué E: Normal and leukemic stem cell niches: Insights and therapeutic opportunities. Cell Stem Cell 16: 254-267, 2015.

32. Hanoun M, Zhang D, Mizoguchi T, Pinho S, Pierce H, Kunisaki Y, Lacombe J, Armstrong SA, Dührsen U and Frenette PS: Acute myelogenous leukemia-induced sympathetic neuropathy promotes malignancy in an altered hematopoietic stem cell niche. Cell Stem Cell 15: 365-375, 2014.

33. Jurczyszyn A, Czepiel J, Gdula-Argasińska J, Perucki W, Skotnicki AB and Majka M: The analysis of the relationship between multiple myeloma cells and their microenvironment. J Cancer 6: 160-168, 2015.

34. Sperling AS, Gibson CJ and Ebert BL: The genetics of myelodysplastic syndrome: From clonal haematopoiesis to secondary leukaemia. Nat Rev Cancer 17: 5-19, 2017.

35. Jootar S, Pornprasertsud N, Petvises S, Rerkamnuaychoke B, Disthabanchong S, Pakakasama S, Ungkanont A and Hongeng S: Bone marrow derived mesenchymal stem cells from chronic myeloid leukemia $t(9 ; 22)$ patients are devoid of Philadelphia chromosome and support cord blood stem cell expansion. Leuk Res 30: 1493-1498, 2006.

36. Wöhrer S, Rabitsch W, Shehata M, Kondo R, Esterbauer H, Streubel B, Sillaber C, Raderer M, Jaeger U, Zielinski C and Valent P: Mesenchymal stem cells in patients with chronic myelogenous leukaemia or bi-phenotypic $\mathrm{Ph}+$ acute leukaemia are not related to the leukaemic clone. Anticancer Res 27: 3837-3841, 2007.
37. Hamidi T, Singh AK and Chen T: Genetic alterations of DNA methylation machinery in human diseases. Epigenomics 7: 247-265, 2015.

38. Akhavan-Niaki $\mathrm{H}$ and Samadani AA: DNA methylation and cancer development: Molecular mechanism. Cell Biochem Biophys 67: 501-513, 2013.

39. Weckselblatt $B$ and Rudd MK: Human structural variation: Mechanisms of chromosome rearrangements. Trends Genet 31: 587-599, 2015.

40. University of Chicago Hematopoietic Malignancies Cancer Risk Team: How I diagnose and manage individuals at risk for inherited myeloid malignancies. Blood 128: 1800-1813, 2016.

41. Ben-David U, Mayshar Y and Benvenisty N: Large-scale analysis reveals acquisition of lineage-specific chromosomal aberrations in human adult stem cells. Cell Stem Cell 9: 97-102, 2011.

This work is licensed under a Creative Commons

Attribution-NonCommercial-NoDerivatives 4.0 International (CC BY-NC-ND 4.0) License. 